\title{
Quantum Measurements Performed with a Single-Electron Transistor
}

\author{
Alexander Shnirman and Gerd Schön \\ Institut für Theoretische Festkörperphysik, Universität Karlsruhe, D-76128 Karlsruhe, Germany
}

\begin{abstract}
Low-capacitance Josephson junction systems as well as coupled quantum dots, in a parameter range where single charges can be controlled, provide physical realizations of quantum bits, discussed in connection with quantum computing. The necessary manipulation of the quantum states can be controlled by applied gate voltages. In addition, the state of the system has to be read out. Here we suggest to measure the quantum state by coupling a single-electron transistor to the $\mathrm{q}-\mathrm{bit}$. As long as no transport voltage is applied, the transistor influences the quantum dynamics of the q-bit only weakly. We have analyzed the time evolution of the density matrix of the transistor and q-bit when a voltage is turned on. For values of the capacitances and temperatures which can be realized by modern nano-techniques the process constitutes a quantum measurement process.
\end{abstract}

\section{INTRODUCTION}

Recent proposals ${ }^{1-4}$ suggested to use nanoscale devices, such as low-capacitance Josephson junctions or coupled quantum dots as quantum bits (q-bits), which are the basic elements of quantum computers. The two logical states are different charge states of the system ${ }^{1-3}$. Applied gate voltages allow the necessary controlled manipulations (single-bit and two-bit operations) of the quantum states. In addition to these manipulations, a read-out device is required to perform quantum measurements of the resulting state of the q-bit. We suggest to use single-electron transistors for this purpose.

The requirements to perform, on one hand, quantum manipulations and, on the other hand, a quantum measurement appear to contradict each other. During the manipulations the dephasing should be minimized, while a quantum measurement should dephase the state of the q-bit as fast as possible. The option to couple the measuring device to the q-bit only when needed is hard to achieve in mesoscopic systems. The alternative, which we discuss here, is to keep the measuring device permanently coupled to the q-bit in a state of equilibrium during the quantum operations. The measurement is performed by driving the measuring device out of equilibrium, in a way which dephases the quantum state of the q-bit. Similar nonequilibrium dephasing processes have recently been considered by a number of authors ${ }^{5-8}$

For definiteness we discuss in this paper the measurement process performed by a single-electron tunneling (SET) transistor coupled capacitively to a Josephson junction q-bit; however, this type of measurements may be performed for any quantum system with two different charge states. We describe the measuring process by considering the timeevolution of the density matrix of the coupled system. We show that the process is characterized by three different time scales: the dephasing time, the time of measurement, which may be longer than the dephasing time, and the mixing time, i.e. the time after which all the information about the initial quantum state is lost due to the transitions induced by the measurement. Thus, we arrive at a new criterion for a "good" quantum measurement: the mixing time should be longer than the time of measurement.

\section{THE QUANTUM SYSTEM AND THE MEASURING DEVICE}

The system is shown in Fig. 1. The two superconducting islands in the upper part are the realization of a q-bit. Its state is characterized by a discrete variable, $n$, the number of extra Cooper pairs on the lower superconducting island. The lower part (a normal island between two normal leads) stands for a SET transistor, which is coupled capacitively to the q-bit. It's charging state is characterized by the extra charge on the middle island, $e N$. A similar setup has recently been studied in the experiments of Refs. [9, 10] with the purpose to demonstrate that the ground state of a single Cooper pair box is a coherent superposition of different charge states. We discuss the relation of our proposal to these experiments below.

As shown earlier ${ }^{1}$ the quantum operations with the $\mathrm{q}^{-b i t}$ are performed by controlling the applied gate voltage $V_{\mathrm{qb}}$. At this stage the transport voltage $V$ across the SET transistor is kept zero. Therefore no dissipative currents flow in the system, and dephasing effects due to the transistor are minimized. To perform a measurement one applies a transport voltage $V$. The resulting normal current through the transistor depends on the charge configuration of the $\mathrm{q}$-bit, since different charge states induce different voltages on the middle island of the SET transistor. In order to check whether the dissipative current through the SET transistor contains information about the quantum state of 
the q-bit, we have to discuss various noise factors (shot noise) and the measurement induced transitions between the states of the q-bit. It turns out that for suitable parameters, which can be realized experimentally, the dephasing by the passive SET transistor is weak. When the transport voltage is turned on the dephasing is fast, and the current through the transistor - after a transient period - provides a measure of the state of the q-bit. At still longer times the complicated dynamics of the composite system destroys the information of the quantum state to be measured.

The Hamiltonian of the composite system consists of three main parts: the charging energy, the terms describing the microscopic degrees of freedom of the metal islands and electrodes, and the tunneling terms, including the Josephson coupling. The charging term is a quadratic form in the variables $n$ and $N$ :

$$
\begin{aligned}
H_{\text {charge }}= & E_{\mathrm{qb}} n^{2}+E_{\mathrm{set}} N^{2}+E_{\mathrm{int}} n N+ \\
& 2 e n V_{n}+e N V_{N}+\text { const. }
\end{aligned}
$$

The charging energy scales $E_{\mathrm{qb}}, E_{\mathrm{set}}$ and $E_{\text {int }}$ are determined by the capacitances between all the islands. Similarly, the effective gate voltages $V_{n}$ and $V_{N}$ depend in general on all three voltages $V_{\mathrm{qb}}, V_{g}$ and $V$, but for a symmetric bias (see Fig. 1), $V_{n}$ and $V_{N}$ are controlled only by the two gate voltages, $V_{\mathrm{qb}}$ and $V_{g}$.

The microscopic terms $H_{\mathrm{L}}, H_{\mathrm{R}}$ and $H_{\mathrm{I}}$ describe noninteracting electrons in the two leads and on the middle island of the SET transistor, respectively:

$$
H_{r}=\sum_{k \sigma} \epsilon_{k \sigma}^{r} c_{k \sigma}^{r \dagger} c_{k \sigma}^{r} \quad(r=\mathrm{L}, \mathrm{R}, \mathrm{I})
$$

The index $\sigma$ labels the transverse channels including the spin, while $k$ labels the wave vector within one channel. Similar terms exist for the two islands of the q-bit. Here we use the "macroscopic" description of the superconductors, assuming that the microscopic degrees of freedom have already been integrated out ${ }^{11}$.

The tunneling terms include the Josephson coupling $H_{\mathbf{J}}=-E_{\mathbf{J}} \cos \Theta$, which describes the transfer of Cooper pairs between the two islands of the $\mathrm{q}$-bit $\left(e^{i \Theta}|n\rangle=|n+1\rangle\right)$, and the normal tunneling Hamiltonian for the SET transistor:

$$
H_{\mathrm{T}}=\sum_{k k^{\prime} \sigma} T_{k k^{\prime} \sigma}^{L} c_{k \sigma}^{\mathrm{L \dagger}} c_{k^{\prime} \sigma}^{\mathrm{I}} e^{-i \phi}+\sum_{k^{\prime} k^{\prime \prime} \sigma} T_{k^{\prime} k^{\prime \prime} \sigma}^{R} c_{k^{\prime \prime} \sigma}^{\mathrm{R} \dagger} c_{k^{\prime} \sigma}^{\mathrm{I}} e^{-i \phi} e^{i \psi}+\text { h.c. }
$$

Apart from the microscopic degrees of freedom, (3) contains two "macroscopic" operators: $e^{ \pm i \phi}$ and $e^{ \pm i \psi}$. The first one describes changes of the charge on the transistor island due to the tunneling: $e^{i \phi}|N\rangle=|N+1\rangle$. It may be treated as an independent degree of freedom if the total number of electrons on the island is large. We include one more operator $e^{ \pm i \psi}$ which describes the changes of the charge in the right lead. It acts on $m$, the number of electrons which have tunneled through the SET transistor, $e^{i \psi}|m\rangle=|m+1\rangle$. Since the chemical potential of the right lead is controlled, $m$ does not appear in any charging part of the Hamiltonian. However, $e^{ \pm i \psi}$ allows us to keep track of the number of electrons which have passed through the SET transistor, which is related to the current through the device.

We define the q-bit's Hamiltonian as the part of the total one which governs the q-bit's dynamics in equilibrium $(N=0)$ :

$$
H_{\mathrm{qb}}=E_{\mathrm{qb}}\left(n-Q_{\mathrm{qb}}\right)^{2}-E_{\mathbf{J}} \cos \Theta
$$

Here $Q_{\mathrm{qb}} \equiv-2 e V_{n} / 2 E_{\mathrm{qb}}$ is the q-bit's gate charge, measured in units of $2 e$. We concentrate on the values of $Q_{\mathrm{qb}}$ in an interval around the degeneracy point $Q_{\mathrm{qb}}=1 / 2$, so that only the low energy charge states $n=0$ and $n=1$ are relevant. These states, however, are not appropriate logical states of the q-bit since they are not the eigenstates of the Hamiltonian (4). We diagonalize (4) in the two charge states subspace for a fixed value of $Q_{\mathrm{qb}}$ (which is kept constant between the quantum manipulations and during the measurement) and denote the corresponding logical states $|0\rangle$ and $|1\rangle$. In the new basis, up to a constant, $H_{\mathrm{qb}}=-(1 / 2) \Delta E \sigma_{z}$, where $\sigma_{z}$ is the Pauli matrix and

$$
\Delta E \equiv \sqrt{\left[E_{\mathrm{qb}}\left(1-2 Q_{\mathrm{qb}}\right)\right]^{2}+E_{\mathbf{J}}^{2}}
$$

The price which we pay for this simplification is that the number operator $n$, which appears in the mixed term of (1), becomes non-diagonal:

$$
n=\frac{1}{2}-\frac{1}{2} \cos \eta \sigma_{z}-\frac{1}{2} \sin \eta \sigma_{x}
$$

with mixing angle $\eta$ given by $\tan \eta=E_{\mathbf{J}} / E_{\mathrm{qb}}\left(1-2 Q_{\mathrm{qb}}\right)$. In the quantum regime, which we are considering here, $E_{\mathrm{qb}} \gg E_{\mathbf{J}}$ and, therefore, one can choose $Q_{\mathrm{qb}}$ so that $\tan \eta \ll 1$. 
The interaction Hamiltonian (part of the mixed term in (1)) now becomes

$$
H_{\mathrm{int}}=-\frac{1}{2} E_{\mathrm{int}} N\left(\cos \eta \sigma_{z}+\sin \eta \sigma_{x}\right)
$$

while the rest of the mixed term $\left(E_{\text {int }} N / 2\right)$ as well as all other remaining terms are collected in the Hamiltonian of the SET transistor:

$$
H_{\text {set }}=E_{\text {set }}\left(N-Q_{\text {set }}\right)^{2}+H_{\mathrm{L}}+H_{\mathrm{R}}+H_{\mathrm{I}}+H_{\mathrm{T}} \text {. }
$$

The transistor's gate charge (measured in the units of $e$ ) became $Q_{\text {set }} \equiv-\left(e V_{N}+E_{\text {int }} / 2\right) / 2 E_{\text {set }}$. The total Hamiltonian reads $H=H_{\mathrm{qb}}+H_{\mathrm{set}}+H_{\mathrm{int}}$. One should understand, however, that the division chosen is rather arbitrary. The terms $H_{\mathrm{qb}}$ and $H_{\text {set }}$ would not describe the q-bit and the SET transistor if they were decoupled.

\section{QUANTITATIVE DESCRIPTION OF THE MEASUREMENT, TIME EVOLUTION OF THE REDUCED DENSITY MATRIX}

The total system is described by a reduced density matrix $\sigma(t)=\operatorname{Tr}_{\mathrm{L}, \mathrm{R}, \mathrm{I}}\{\rho(t)\}$, where the trace is taken over the microscopic states of the leads and of the island. In general, the density matrix $\sigma\left(i, j ; N, N^{\prime} ; m, m^{\prime}\right)$ is a matrix in $i, j$, which stand, for the quantum states of the q-bit $(|0\rangle$ or $|1\rangle)$, in $N$, and in $m$. However, as has been shown in ${ }^{12}$ a closed set of equations describing the time evolution of the system can be derived where the off-diagonal elements in $N$ have been eliminated. The same is true for the off-diagonal elements in $m$. Therefore, we need to consider only the following elements of the density matrix $\sigma_{i, j}^{N, m} \equiv \sigma(i, j ; N, N ; m, m)$. We assume now that at time $t=0$, when the q-bit is prepared in the quantum state $a|0\rangle+b|1\rangle$ as a result of previous quantum manipulations, we switch on a transport voltage to the SET transistor. To proceed we can further reduce the density matrix in two different ways to obtain dual descriptions of the measuring process.

The first widely used procedure ${ }^{7}$ is to trace over $N$ and $m$. This yields a reduced density matrix of the q-bit $\sigma_{i, j} \equiv \sum_{N, m} \sigma_{i, j}^{N, m}$. Assuming that at $t=0$ it is in the state

$$
\sigma_{i, j}(0)=\left(\begin{array}{cc}
|a|^{2} & a b^{*} \\
a^{*} b & |b|^{2}
\end{array}\right),
$$

the questions are how fast the off-diagonal elements of $\sigma_{i, j}$ vanish (dephasing), and how fast the diagonal elements change their original values (for instance due to transitions induced by the measurement). This description is enough when one is interested in the quantum properties of the measured system only (q-bit in our case) and the measuring device is used as a source of dephasing ${ }^{5,6,8}$. It does not tell us much, however, about the quantity measured in an experiment, namely the current flowing trough the SET transistor.

The second procedure is to evaluate the probability distribution of the number of electrons $m$ which have tunneled trough the SET transistor during time $t$ :

$$
P(m, t) \equiv \sum_{N, i} \sigma_{i, i}^{N, m}(t) .
$$

This quantity gives a complete description of the measurement. At $t=0$ no electrons have tunneled, so $P(m, 0)=\delta_{m, 0}$. Then this delta-peak starts to shift in positive $m$ direction and, at the same time, it widens due to shot noise. Since two states of the q-bit correspond to different conductivities (and shift velocities in $m$ space), one may hope that after some time the peak splits into two. If after sufficient separation of the two peaks their weights (integrals) are still close to $|a|^{2}$ and $|b|^{2}$, a good quantum measurement has been performed. Unfortunately, there exist further processes which destroy this idealized picture. After a long time the two peaks transform into a broad plateau, since transitions between the q-bit's states are induced by the measurement. Therefore, one should find an optimum time for the measurement, so that, on one hand, the two peaks are separate and, on the other hand, the induced transitions have not yet happened. In order to describe this we have to analyze the time evolution of the reduced density matrix quantitatively.

\section{DERIVATION OF THE MASTER EQUATION}

The Bloch-type or master equations with coherent terms have only recently been analyzed in the condensed matter physics $^{13,7}$. In Ref. [ 12] a diagrammatic technique has been developed which provides a formally exact master 
equation as an expansion in the tunneling strength. Only the tunneling term $H_{\mathrm{T}}$ is considered a perturbation, while all other terms constitute the zeroth order Hamiltonian $H_{0} \equiv H-H_{\mathrm{T}}$, which is treated exactly. The master equation reads

$$
\frac{d \sigma(t)}{d t}-\frac{i}{\hbar}\left[\sigma(t), H_{0}\right]=\int_{0}^{t} d t^{\prime} \Sigma\left(t-t^{\prime}\right) \sigma\left(t^{\prime}\right),
$$

where the matrix elements of $\Sigma\left(t^{\prime}, t\right)$ can be calculated diagrammatically using the real-time Keldysh contour technique $^{12}$. The simplest diagram describing the tunneling through the left junction in first order perturbation theory (sequential tunneling) is shown in Fig. 2. The dashed lines crossing the diagram contribute the following factor to the rate ${ }^{12}$ :

$$
-\alpha_{\mathrm{L}} \frac{\left(\frac{\pi}{\hbar \beta}\right)^{2} e^{ \pm i \mu_{\mathrm{L}}\left(t-t^{\prime}\right)}}{\sinh ^{2}\left[\frac{\pi}{\hbar \beta}\left(t-t^{\prime} \pm i \delta\right)\right]}
$$

where $\alpha_{\mathrm{L}} \equiv \hbar /\left(4 \pi^{2} e^{2} R_{\mathrm{T}_{\mathrm{L}}}\right), \mu_{\mathrm{L}}$ is the electro-chemical potential of the left lead, and $\delta \equiv 1 / \omega_{c}$ is the inverse frequency cut-off. The sign of the $i \delta$ term depends on the time-direction of the dashed line ${ }^{12}$. It is minus if the direction of the line with respect to the Keldysh contour coincides with its direction with respect to the absolute time (from left to right), and plus otherwise. For example the right part of Fig. 2 should carry a minus sign, while the left part carries a plus sign. For the sign in front of $i \mu_{\mathrm{L}}\left(t-t^{\prime}\right)$ the rule is as follows: minus, if the line goes forward with respect to the absolute time, and plus otherwise.

For a single SET transistor the horizontal lines correspond to trivial exponential factors ${ }^{12} e^{i E t}$. In our case, however, we have to account for the nontrivial time evolution of the q-bit. Therefore the upper line in the left part of Fig. 2 corresponds to $\left\langle N-1, j\left|e^{-i H_{0}\left(t-t^{\prime}\right)}\right| N-1, j^{\prime}\right\rangle$, while the lower line corresponds to $\left\langle N, i\left|e^{i H_{0}\left(t-t^{\prime}\right\rangle}\right| N, i^{\prime}\right\rangle$. To calculate these matrix elements we diagonalize $H_{\mathrm{qb}}+H_{\mathrm{int}}$ for each value of $N$. The eigenenergies are

$$
E_{0,1}^{(N)}=\mp \frac{1}{2} \sqrt{\left(\Delta E+E_{\text {int }} N \cos \eta\right)^{2}+\left(E_{\text {int }} N \sin \eta\right)^{2}}
$$

and the mixing angles $\epsilon_{N}$ (analogous to $\eta$ ) are given by $\tan \epsilon_{N}=E_{\text {int }} N \sin \eta /\left(\Delta E+E_{\text {int }} N \cos \eta\right)$. The matrix elements (propagators) read:

$$
\begin{aligned}
& \left\langle N, 0\left|e^{-i H_{0} \Delta t}\right| N, 0\right\rangle=\left(\cos ^{2} \frac{\epsilon_{N}}{2} e^{-i E_{0}^{N} \Delta t}+\sin ^{2} \frac{\epsilon_{N}}{2} e^{-i E_{1}^{N} \Delta t}\right) e^{-i E_{\mathrm{set}}^{(N)} \Delta t} \\
& \left\langle N, 1\left|e^{-i H_{0} \Delta t}\right| N, 1\right\rangle=\left(\cos ^{2} \frac{\epsilon_{N}}{2} e^{-i E_{1}^{N} \Delta t}+\sin ^{2} \frac{\epsilon_{N}}{2} e^{-i E_{0}^{N} \Delta t}\right) e^{-i E_{\mathrm{set}}^{(N)} \Delta t} \\
& \left\langle N, 1\left|e^{-i H_{0} \Delta t}\right| N, 0\right\rangle=\frac{1}{2} \sin \epsilon_{N}\left(e^{-i E_{0}^{N} \Delta t}-e^{-i E_{1}^{N} \Delta t}\right) e^{-i E_{\mathrm{set}}^{(N)} \Delta t}
\end{aligned}
$$

where $E_{\text {set }}^{(N)} \equiv E_{\text {set }}\left(N-Q_{\text {set }}\right)^{2}$.

We analyze now the rates in Fig. 2 for different choices of q-bit's indices in the regime $\Delta E \gg E_{\text {int }}, E_{\mathbf{J}}$. There the mixing angles are small, $\epsilon_{N} \propto N E_{\text {int }} E_{\mathbf{J}} /(\Delta E)^{2}$, for all relevant values of $N$. Hence, we keep only terms linear in $\epsilon_{N}$. The simplest transition $\left(i^{\prime}=0, j^{\prime}=0, N-1, m\right) \rightarrow(i=0, j=0, N, m)$ is described by

$$
\Sigma_{\substack{(1) \\ N-1, m, 0 ; N, m, 0}}^{N-1, m, 0 ; N, m, 0}(\Delta t)=\frac{-\alpha_{\mathrm{L}}\left(\frac{\pi}{\hbar \beta}\right)^{2} e^{-i \tilde{E} \Delta t}}{\sinh ^{2}\left[\frac{\pi}{\hbar \beta}(\Delta t+i \delta)\right]}+c . c .
$$

where $\tilde{E}$ stands here for $\mu_{\mathrm{L}}+\left(E_{\text {set }}^{(N-1)}-E_{\text {set }}^{(N)}\right)+\left(E_{0}^{N-1}-E_{0}^{N}\right)$.

The form of the master equation (11) suggests the use of a Laplace transformation, after which the last term in (11) becomes $\Sigma(s) \sigma(s)$. We Laplace transform (15) in the regime $s \ll \tilde{E}$, i.e. we assume the density matrix $\sigma$ to change slowly on the time scale given by $\hbar / \tilde{E}$. This assumption should be verified later for self-consistency. At zero temperature $(\beta \rightarrow \infty)$ and for $\delta \rightarrow 0$ we obtain:

$$
\begin{aligned}
& \Sigma^{(1)} \begin{array}{l}
N-1, m, 0 ; N, m, 0 \\
N-1, m, 0 ; N, m, 0
\end{array}(s)=2 \alpha_{\mathrm{L}} \operatorname{Re}\left[(s+i \tilde{E}) e^{i \delta(s+i \tilde{E})} E_{1}[i \delta(s+i \tilde{E})]\right] \\
& \approx 2 \pi \alpha_{\mathrm{L}} \tilde{E} \Theta(\tilde{E})-2 \alpha_{\mathrm{L}} s(1+\gamma+\ln (|\delta \tilde{E}|))
\end{aligned}
$$

where $E_{1}[\ldots]$ is the exponential integral and $\gamma \approx 0.58$ is Euler's constant. Denoting the diverging factor $[1+\gamma+\ln (|\delta \tilde{E}|)]$ by $D(\tilde{E})$ and performing the inverse Laplace transform we arrive at: 
(Note that (17) is equivalent to (15) only as a kernel in the convolution (11) when applied to slowly changing matrix elements of $\sigma$ ). The first term of (17) is the usual Golden Rule tunneling rate corrected with respect to the additional charging energy corresponding to the quantum state $|0\rangle$ of the q-bit, $E_{0}^{N-1}-E_{0}^{N}$. The second (diverging) part of (17) produces a term proportional to $\frac{d}{d t} \sigma_{0,0}^{N-1, m}$. One can take this term to the LHS of (11) so that the time derivative in the LHS will look like $\frac{d}{d t}\left[\sigma_{0,0}^{N, m}-2 \alpha_{\mathrm{L}} D(\tilde{E}) \sigma_{0,0}^{N-1, m}\right]$. We analyze all possible choices of the q-bit's indices in Fig. 2 and arrive at the conclusion that the diverging terms have always the same structure as the coherent terms in the LHS of (11). Moreover, if we neglect some energy corrections of order $E_{\text {int }}$, we may incorporate all of these terms to the LHS of (11), so that the master equation reads:

$$
\left(1+\alpha_{\mathrm{L}} A+\alpha_{\mathrm{R}} B\right)\left[\frac{d \sigma(t)}{d t}-\frac{i}{\hbar}\left[\sigma(t), H_{0}\right]\right]=\Gamma \sigma(t)
$$

where $A$ and $B$ are three-diagonal matrices in the $N$ and $m$ spaces, composed of the diverging factors of the type of $D(\tilde{E})$, while $\Gamma$ is the regular local part of $\Sigma\left(t-t^{\prime}\right)$.

We expect that without the approximation of energies in the diverging terms the structure of (18) would be the same, with $A$ and $B$ being more complicated matrices, which would include some mixing in the space of the q-bit's states. Finally, we note that for any physically reasonable choice of the cut-off $\delta$, the logarithmically divergent factors in the matrices $A$ and $B$ are of order one, and, therefore, the mixing corrections to the unit matrix in the LHS of (18) are small. We multiply the master equation (18) by $\left(1+\alpha_{\mathrm{L}} A+\alpha_{\mathrm{R}} B\right)^{-1} \approx\left(1-\alpha_{\mathrm{L}} A-\alpha_{\mathrm{R}} B\right)$ from the left, so that the mixing corrections move to the RHS. Since $\Gamma$ is linear in $\alpha_{\mathrm{L}}$ and $\alpha_{\mathrm{R}}$, the mixing corrections are quadratic. We drop them in the framework of the first order perturbation theory. The master equation to be analyzed thus becomes:

$$
\frac{d \sigma(t)}{d t}-\frac{i}{\hbar}\left[\sigma(t), H_{0}\right]=\Gamma \sigma(t)
$$

If the applied voltage is not too high (the exact criterion to be specified) we may consider only two charge states of the SET transistor, $N=0,1$. We perform a Fourier transform in $m$ space $\sigma_{i, j}^{N}(k) \equiv \sum_{m} \sigma_{i, j}^{N, m} e^{i k m}$. To shorten formulas we introduce $A^{N} \equiv \sigma_{0,0}^{N}(k), B^{N} \equiv \sigma_{1,1}^{N}(k), C^{N} \equiv \sum_{m} \operatorname{Re} \sigma_{0,1}^{N, m} e^{i k m}$, and $D^{N} \equiv \sum_{m} \operatorname{Im} \sigma_{0,1}^{N, m} e^{i k m}$. This enables us to rewrite (19) as:

$$
\begin{aligned}
& \dot{A}^{0}=-\Gamma_{\mathrm{L}_{0}} A^{0}+\Gamma_{\mathrm{R}_{0}} e^{i k} A^{1}-\omega_{\mathrm{L}} C^{0}-\omega_{\mathrm{R}} e^{i k} C^{1} \\
& \dot{A}^{1}=\Gamma_{\mathrm{L}_{0}} A^{0}-\Gamma_{\mathrm{R}_{0}} A^{1}-\Omega D^{1}+\omega_{\mathrm{L}} C^{0}+\omega_{\mathrm{R}} C^{1} \\
& \dot{B}^{0}=-\Gamma_{\mathrm{L}_{1}} B^{0}+\Gamma_{\mathrm{R}_{1}} e^{i k} B^{1}-\omega_{\mathrm{L}} C^{0}-\omega_{\mathrm{R}} e^{i k} C^{1} \\
& \dot{B}^{1}=\Gamma_{\mathrm{L}_{1}} B^{0}-\Gamma_{\mathrm{R}_{1}} B^{1}+\Omega D^{1}+\omega_{\mathrm{L}} C^{0}+\omega_{\mathrm{R}} C^{1} \\
& \dot{C}^{0}=-\Delta E^{0} D^{0}-\Gamma_{L} C^{0}+\Gamma_{R} e^{i k} C^{1}-\frac{\omega_{\mathrm{L}}}{2}\left(A^{0}-B^{0}\right)-\frac{\omega_{\mathrm{R}}}{2} e^{i k}\left(A^{1}-B^{1}\right) \\
& \dot{C}^{1}=-\Delta E^{1} D^{1}+\Gamma_{L} C^{0}-\Gamma_{R} C^{1}+\frac{\omega_{\mathrm{L}}}{2}\left(A^{0}-B^{0}\right)+\frac{\omega_{\mathrm{R}}}{2}\left(A^{1}-B^{1}\right) \\
& \dot{D}^{0}=\Delta E^{0} C^{0}-\Gamma_{L} D^{0}+\Gamma_{R} e^{i k} D^{1} \\
& \dot{D}^{1}=\Delta E^{1} C^{1}+\Gamma_{L} D^{0}-\Gamma_{R} D^{1}+\frac{\Omega}{2}\left(A^{1}-B^{1}\right) .
\end{aligned}
$$

Here $\Delta E^{0,1} \equiv E_{1}^{0,1}-E_{0}^{0,1}$ are the energy differences between the q-bit's states for $N=0$ and $N=1$ respectively, and $\Omega \equiv E_{\mathrm{int}} \sin \eta$ is the coefficient in the mixing term in $H_{\text {int }}$ for $N=1$ (see ( 7 )). The terms proportional to $\Delta E^{0,1}$ and $\Omega$ originate from the coherent part of (19). The tunneling rates which appear in the four last equations for the off-diagonal elements (24-27) are given by

$$
\begin{aligned}
& \Gamma_{L} \equiv 2 \pi \alpha_{\mathrm{L}}\left[\mu_{\mathrm{L}}-E_{\mathrm{set}}\left(1-2 Q_{\mathrm{set}}\right)\right] \\
& \Gamma_{R} \equiv 2 \pi \alpha_{\mathrm{R}}\left[-\mu_{\mathrm{R}}+E_{\mathrm{set}}\left(1-2 Q_{\mathrm{set}}\right)\right]
\end{aligned}
$$

The rates

$$
\begin{aligned}
\Gamma_{L_{0,1}} & \equiv \Gamma_{L} \pm \Delta \Gamma_{L} \\
\Gamma_{\mathrm{R}_{0,1}} & \equiv \Gamma_{R} \pm \Delta \Gamma_{R}
\end{aligned}
$$


appearing in the equations for the diagonal elements (20-23) are corrected due to the charging energy induced by the q-bit:

$$
\begin{aligned}
& \Delta \Gamma_{\mathrm{L}} \equiv 2 \pi \alpha_{\mathrm{L}}\left(E_{0}^{0}-E_{0}^{1}\right) \\
& \Delta \Gamma_{\mathrm{R}} \equiv-2 \pi \alpha_{\mathrm{R}}\left(E_{0}^{0}-E_{0}^{1}\right) .
\end{aligned}
$$

These correction are, actually, responsible for the separation of the peaks. In the regime tan $\eta \ll 1$, which we assume here, $\left|\Delta \Gamma_{\mathrm{L}, \mathrm{R}}\right| \approx 2 \pi \alpha_{\mathrm{L}, \mathrm{R}} E_{\text {int }}$. The rest are small mixing terms, $\omega_{\mathrm{L}, \mathrm{R}} \equiv \pi \alpha_{\mathrm{L}, \mathrm{R}} \sin \epsilon_{1} \Delta E^{1}$, which also originate from diagrams of the type in Fig. 2. Note, that we assume that only the two rates given in (28) are nonzero (two-state approximation). Moreover we assume that the q-bit's charging energy corrections can at most change these two rates, but they can not switch on any other rate or switch off one of the two in (28).

\section{QUALITATIVE ANALYSIS OF THE MASTER EQUATION}

First, we analyze the system (20-27) qualitatively. Imagine that we can "switch off" the Josephson coupling during the measurement. Then all the mixing terms in $(20-27)$, i.e. those proportional to $\Omega$ and $\omega_{\mathrm{L}, \mathrm{R}}$ disappear, and the system factorizes into three independent groups. The first one $(20,21)$,

$$
\begin{aligned}
& \dot{A}^{0}=-\Gamma_{\mathrm{L}_{0}} A^{0}+\Gamma_{\mathrm{R}_{0}} e^{i k} A^{1} \\
& \dot{A}^{1}=\Gamma_{\mathrm{L}_{0}} A^{0}-\Gamma_{\mathrm{R}_{0}} A^{1}
\end{aligned}
$$

has plain wave solutions $\left(\propto e^{i \omega t}\right)$. The standard analysis gives for eigenvalues:

$$
\omega_{1,2}=\frac{i}{2}\left(\Gamma_{\mathrm{L}_{0}}+\Gamma_{\mathrm{R}_{0}}\right)\left\{1 \pm\left[1+\frac{4 \Gamma_{0}\left(e^{i k}-1\right)}{\Gamma_{\mathrm{L}_{0}}+\Gamma_{\mathrm{R}_{0}}}\right]^{\frac{1}{2}}\right\},
$$

where

$$
\Gamma_{0} \equiv \frac{\Gamma_{\mathrm{L}_{0}} \Gamma_{\mathrm{R}_{0}}}{\Gamma_{\mathrm{L}_{0}}+\Gamma_{\mathrm{R}_{0}}}
$$

is the total transport rate corresponding to the $\mathrm{q}$-bit is in the state $|0\rangle$. When $k$ is small $\omega_{1} \approx i\left(\Gamma_{\mathrm{L}_{0}}+\Gamma_{\mathrm{R}_{0}}\right)$, while $\omega_{2} \approx \Gamma_{0} k+i \Gamma_{0}\left(1+\frac{2 \Gamma_{0}}{\Gamma_{\mathrm{L}_{0}}+\Gamma_{\mathrm{R}_{0}}}\right) k^{2}$. Since $\omega_{1}$ is a large imaginary number, already after a short time, $1 /\left|\omega_{1}\right|$, only the second eigenvector $\left(A^{1} / A^{0}=\Gamma_{\mathrm{L}_{0}} / \Gamma_{\mathrm{R}_{0}}\right)$ survives. This eigenvector multiplies a wave packet propagating with the group velocity $\Gamma_{0}$. The wave packet widens due to shot noise of the single electron tunneling, it's width being given by $\sqrt{\Gamma_{0} t}$ (the second imaginary term in the expression for $\omega_{2}$ ).

Analogously the second group of equations $(22,23)$ gives a wave packet with the group velocity $\Gamma_{1} \equiv \frac{\Gamma_{L_{1}} \Gamma_{\mathrm{R}_{1}}}{\left(\Gamma_{\mathrm{L}_{1}}+\Gamma_{\mathrm{R}_{1}}\right)}$ and the width $\approx \sqrt{\Gamma_{1} t}$. The two peaks correspond to the q-bit in the states $|0\rangle$ and $|1\rangle$, respectively. They separate when their width is smaller then the distance between their centers $\sqrt{\Gamma_{0} t}+\sqrt{\Gamma_{1} t} \leq\left|\Gamma_{0}-\Gamma_{1}\right| t$. After this time,

$$
t_{\mathrm{ms}} \equiv\left|\sqrt{\Gamma_{0}}-\sqrt{\Gamma_{1}}\right|^{-2}
$$

which we denote as the measurement time, the process can constitute a quantum measurement. Similar expressions have been obtained in Refs. [5-7], where they have been denoted as dephasing time.

To get a clue for the dephasing we analyze the third group of equations (24-27) at $k=0$ (the trace over $m$ is equivalent to $k=0$ ). These equations may be combined into two complex ones:

$$
\begin{aligned}
& \dot{\sigma}_{0,1}^{0}=i \Delta E^{0} \sigma_{0,1}^{0}-\Gamma_{L} \sigma_{0,1}^{0}+\Gamma_{R} \sigma_{0,1}^{1} \\
& \dot{\sigma}_{0,1}^{1}=i \Delta E^{1} \sigma_{0,1}^{1}+\Gamma_{L} \sigma_{0,1}^{0}-\Gamma_{R} \sigma_{0,1}^{1}
\end{aligned}
$$

The standard analysis shows that if $d E \equiv\left|\Delta E^{1}-\Delta E^{0}\right| \approx E_{\text {int }} \ll\left(\Gamma_{L}+\Gamma_{R}\right)$ the imaginary parts of the eigenvalues are $\operatorname{Im} \omega_{1} \approx\left(\Gamma_{L}+\Gamma_{R}\right)$ and $\operatorname{Im} \omega_{2} \approx \frac{d E^{2}}{4\left(\Gamma_{L}+\Gamma_{R}\right)}$. In the opposite limit $d E \gg\left(\Gamma_{L}+\Gamma_{R}\right)$ the imaginary parts are $\operatorname{Im} \omega_{1} \approx \Gamma_{L}$ and $\operatorname{Im} \omega_{2} \approx \Gamma_{R}$. The first limit is physically more relevant (we have assumed parameters in this regime), although the second one is also possible if the tunneling is too weak or the coupling between the q-bit ant the SET transistor is too strong. In both limits the dephasing time, which is defined as the the longer of the two times, 


$$
\tau_{\phi} \equiv \max \left\{\left[\operatorname{Im} \omega_{1}\right]^{-1},\left[\operatorname{Im} \omega_{2}\right]^{-1}\right\}
$$

is parametrically different from the measurement time (34). In the first limit, $d E \ll\left(\Gamma_{L}+\Gamma_{R}\right)$, it is

$$
\tau_{\phi}=\frac{4\left(\Gamma_{L}+\Gamma_{R}\right)}{d E^{2}} \propto \alpha_{\mathrm{L}, \mathrm{R}}
$$

while $t_{\mathrm{ms}} \propto \alpha_{\mathrm{L}, \mathrm{R}}^{-1}$. One can check that in the whole range of validity of our approach the measurement time exceeds the dephasing time, $t_{\mathrm{ms}}>\tau_{\phi}$. This is consistent with the fact that a "good" quantum measurement should completely dephase a quantum state. In Refs. [ 5-7], where different systems have been discussed, the expressions for the resulting dephasing time were given by expressions similar to (34), thus $\tau_{\phi}=t_{\mathrm{ms}}$.

In our example the dephasing time is shorter than the measurement time. The reason for this is, probably, the presence of the additional uncontrolled environment provided by the middle island of the SET transistor. The transport of electrons occurs via a real state of the island $N=1$. In different transitions the island may be left in different microscopic states, even though the same number of electrons have passed. To put it in the language of Ref. [14], the initial state of the system $(a|0\rangle+b|1\rangle)|\chi\rangle|m=0\rangle$ evolves into $a|0\rangle\left|\chi_{0}\right\rangle\left|m_{0}\right\rangle+b|1\rangle\left|\chi_{1}\right\rangle\left|m_{1}\right\rangle$, where $|\chi\rangle$ stands for the quantum state of the uncontrolled environment. One may imagine a situation when $m_{0}=m_{1}$, but $\left|\chi_{0}\right\rangle$ and $\left|\chi_{1}\right\rangle$ are orthogonal. In this situation the dephasing has occured but no measurement has been performed.

The additional environment plays, actually, a positive role, i.e. it helps us to perform a quantum measurement, provided it dephases the state of the q-bit only when the system is driven out of equilibrium. This is because the dephasing suppresses the transitions between the states of the q-bit (Zeno effect).

\section{THE MIXING TIME}

Finally, we analyze what happens if we take into account the mixing terms in the system (20-27). We assume $k=0$ and investigate the eigenvalues of the eight by eight matrix formed by the coefficients of (20-27). Note that in the discussion above we have calculated all the eight eigenvalues for $E_{\mathbf{J}}=0$ (the two eigenvalues of the complex system (35) are doubled when one considers it as a system of four real equations). In the diagonal part there were two zeros, which corresponded to two conserved quantities, $A^{0}+A^{1}=\sigma_{0,0}$ and $B^{0}+B^{1}=\sigma_{1,1}$. Six other eigenvalues were large compared to the amplitudes of the mixing terms. It is clear, that switching on the mixing changes only slightly the values of the six large eigenvalues. Moreover, one of the eigenvalues is always zero. This corresponds to the conservation of the total trace $A^{0}+A^{1}+B^{0}+B^{1}=1$. The last (8th) eigenvalue acquires now a small imaginary part and this gives the time scale of the mixing between the two states of the q-bit.

We do not have an analytical expression for the mixing time, but we can estimate it for a concrete physical situation. At the degeneracy point, we have $\Gamma_{\mathrm{L}}=\Gamma_{\mathrm{R}}$, and the corrections to the rates (30) cancel each other, thus, no measurement is performed. Therefore, we choose $Q_{\text {set }}$ far enough from the degeneracy point, which is $Q_{\text {set }}=1 / 2$, so that $\Gamma_{\mathrm{L}}<\Gamma_{\mathrm{R}}$ and the Coulomb blockade energy $E_{\mathrm{CB}} \equiv E_{\mathrm{set}}\left(1-2 Q_{\text {set }}\right)$ is of the order of $E_{\text {set }}$. To satisfy the conditions for the Golden Rule (see (15) and the discussion thereafter) we assume the chemical potential of the left lead $\mu_{\mathrm{L}}=V / 2$ to exceed the Coulomb blockade energy by an amount of the order of $E_{\mathrm{CB}} \propto E_{\text {set }}$ and assume $E_{\mathrm{CB}}$ to be the largest energy scale of the system: $E_{\mathrm{CB}} \gg \Delta E$. The transport voltage should not, however, exceed the limit, after which the third charge state of the SET transistor $N=-1$ becomes involved. Thus $V / 2<E_{\text {set }}\left(1+2 Q_{\text {set }}\right)$ and $Q_{\text {set }}$ should be chosen far enough from zero as well. In this regime we estimate the mixing time as

$$
t_{\mathrm{mix}}^{-1} \propto 2 \pi \alpha \frac{E_{\mathrm{int}}^{2} E_{\mathrm{J}}^{2}}{(\Delta E)^{4}} E_{\mathrm{set}}
$$

where $\alpha_{\mathrm{L}}=\alpha_{\mathrm{R}} \equiv \alpha$. The measurement time in the same regime is given approximately by

$$
t_{\mathrm{ms}}^{-1} \propto 2 \pi \alpha \frac{E_{\mathrm{int}}^{2}}{E_{\mathrm{set}}} .
$$

The exact values of $Q_{\text {set }}$ and $V$ would determine the numerical coefficients in front of (38) and (39). Thus, $t_{\mathrm{ms}} / t_{\mathrm{mix}} \propto$ $E_{\mathbf{J}}^{2} E_{\text {set }}^{2} /(\Delta E)^{4}$. One recognizes two competing ratios here: $E_{\mathbf{J}} / \Delta E$, which is small, and $E_{\mathrm{set}} / \Delta E$, which is large. The condition $t_{\mathrm{ms}} / t_{\mathrm{mix}} \ll 1$, thus, imposes an additional restriction on the parameters of the system. 
To show that all the conditions assumed in this paper are realistic we calculate the charging energies $E_{\mathrm{set}}, E_{\mathrm{qb}}$ and $E_{\text {int }}$ for the following case: the capacitance of the Josephson junction $C_{\mathbf{J}}=2.0 \times 10^{-16} \mathrm{~F}$, the capacitances of the normal junctions $C_{\mathrm{N}}=1.0 \times 10^{-17} \mathrm{~F}$ and the capacitances of all other capacitors $C=2.5 \times 10^{-18} \mathrm{~F}$. We obtain: $E_{\mathrm{set}} \approx 20 \mathrm{~K}$, $E_{\mathrm{qb}} \approx 10 \mathrm{~K}, E_{\mathrm{int}} \approx 0.5 \mathrm{~K}$. Taking $Q_{\mathrm{qb}}=0.35, Q_{\text {set }}=0.15$ and $e V=48 \mathrm{~K}$ we get $\Delta E \approx 3 \mathrm{~K}, E_{\mathrm{CB}} \approx 14 \mathrm{~K}$, and $V / 2-E_{\mathrm{CB}} \approx 10 \mathrm{~K}$. We also assume $2 \pi \alpha=0.1$. The measurement time in this regime is $t_{\mathrm{ms}} \approx 0.38 \times 10^{4} \hbar /\left(k_{\mathrm{B}} 1 \mathrm{~K}\right) \approx$ $0.28 \times 10^{-7} \mathrm{~s}$. For this choice of parameters we calculate $t_{\mathrm{mix}}$ numerically, assuming first $E_{\mathbf{J}}=0.1 \mathrm{~K}$, and we obtain $t_{\text {mix }} \approx 1.4 \times 10^{5} \hbar /\left(k_{\mathrm{B}} 1 \mathrm{~K}\right) \approx 1.0 \times 10^{-6} \mathrm{~s}$. Thus $t_{\mathrm{mix}} / t_{\mathrm{ms}} \approx 35$ and the separation of peaks should occur much earlier than the transitions happen. Indeed, the numerical simulation of the system (20-27) for those parameters given above shows almost ideal separation of peaks (see Fig. 3 ). Then, we calculate $t_{\text {mix }}$ for $E_{\mathbf{J}}=0.25 \mathrm{~K}$, and we obtain $t_{\text {mix }} / t_{\mathrm{ms}} \approx 6$. This is a marginal situation. The numerical simulation in this case shows (see Fig. 4) that the peaks, first, start to separate, but, later, the valley between the peaks is filled due to the transitions.

In this paper we have demonstrated that the current through a single-electron transistor can serve as a measurement of the quantum state of the q-bit, in the sense that in the case of a superposition of two eigenstates it gives one or the other result with the appropriate probabilities. This should be distinguished from another question, namely whether it is possible to demonstrate that an eigenstate of a q-bit can actually be a superposition of two different charge states, i.e. whether it depends on the mixing angle $\eta$ as described by Eq. (6). This question has been addressed in the experiments of Refs. [ 9, 10]. They used a setup similar to the one shown in Fig. 1, a single-Cooper-pair box coupled to a single-electron transistor. They could demonstrate that the expectation, i.e. the average value of the charge in the box varies continuously as a function of the applied gate voltage as follows from (6).

Our theory can also describe the type of measurements performed in Refs. [ 9, 10]. For this purpose we analyze the rates in the master equation (19) for general values of the mixing angle $\eta$, relaxing the requirement tan $\eta \ll 1$. Then, for our approach to be valid, we must have $E_{\text {int }} \ll E_{\mathbf{J}}$, so that $\tan \epsilon_{N} \ll 1$. In this regime each eigenstate of the $\mathrm{q}$-bit, $|0\rangle$ or $|1\rangle$, corresponds to a single, though $\eta$-dependent propagation velocity $\left(\Gamma_{0}\right.$ or $\left.\Gamma_{1}\right)$. Thus, if the $\mathrm{q}^{-\mathrm{bit}}$ is prepared in one of its eigenstates, then even at the degeneracy point $(\eta=\pi / 2)$ where the eigenstates are equally weighted superpositions of two charge states, one would observe only one peak. We have calculated $\Gamma_{0}$ as a function of $\eta$ using (28), (29), (30), and (33) and obtained curves (not shown here) very similar to those in the experiments. It should be added that near degeneracy our setup would not be efficient in projecting onto the eigenstates anymore, since the difference between the velocities of the peaks, $\left|\Gamma_{0}-\Gamma_{1}\right|$, vanishes near the degeneracy point.

To conclude we have shown that a single-electron transistor capacitively coupled to a q-bit may serve as a quantum measuring device in an accessible range of parameters. We have described the process of measurement by deriving the time evolution of the reduced density matrix and we have discussed two dual ways to further reduce it. One way, in which the density matrix of the q-bit is obtained, provides the dephasing time, while the other, in which the number of tunneled electrons is counted, provides the time of measurement. We have shown that, in our case, the dephasing time was shorter than the measurement time, and we have discussed the physical meaning of this result. Finally, we have estimated the mixing time, i.e. the time scale on which the transitions induced by the measurement occur. We have shown that it may be made longer than the measurement time with current technology.

\section{ACKNOWLEDGMENTS}

We thank J. König, Y. Gefen, Y. Levinson, J. E. Mooij, T. Pohjola, H. Schoeller, E. Ben-Jacob, C. Bruder, Y. Makhlin, Z. Hermon for stimulating discussions. This work is supported by the Graduiertenkolleg "Kollektive Phänomene im Festkörper", by the SFB 195 of the DFG, and by the German Israeli Foundation (Contract G-464$247.07 / 95)$.

${ }^{1}$ A. Shnirman, G. Schön, and Z. Hermon, Phys. Rev. Lett. 79, 2371 (1997).

${ }^{2}$ J. E. Mooij, private communication.

${ }^{3}$ D. V. Averin, cond-mat/9706026.

${ }^{4}$ D. Loss and D. P. DiVincenzo, Phys. Rev. A 57, 120 (1998).

${ }^{5}$ Y. Levinson, Europhys. Lett. 39, 299 (1997). 
${ }^{6}$ I. L. Aleiner, N. S. Wingreen, and Y. Meir, Phys. Rev. Lett. 79, 3740 (1997).

${ }^{7}$ S. A. Gurvitz, Phys. Rev. B 56, 15215 (1997); cond-mat/9706074.

${ }^{8}$ E. Buks, R. Schuster, M. Heiblum, D. Mahalu, and V. Umansky, cond-mat/9709022.

${ }^{9}$ V. Bouchiat, P. Joyez, D. Esteve, and M. Devoret, to be published in Physica Scripta.

${ }^{10}$ V. Bouchiat, Ph. D. Thesis, Université Paris 6, (1997).

${ }^{11}$ G. Schön and A. D. Zaikin, Physics Reports 198, 237 (1990).

${ }^{12}$ H. Schoeller and G. Schön, Phys. Rev. B 50, 18436 (1994).

${ }^{13}$ Yu. V. Nazarov, Physica B 189, 57 (1993); T.H. Stoof and Yu. V. Nazarov, Phys. Rev. B 55, 1050 (1996); S. A. Gurvitz and Ya. S. Prager, Phys. Rev. B 53, 15932 (1996).

${ }^{14}$ A. Stern, Y. Aharonov, and Y. Imry, Phys. Rev. A 41, 3436 (1990).

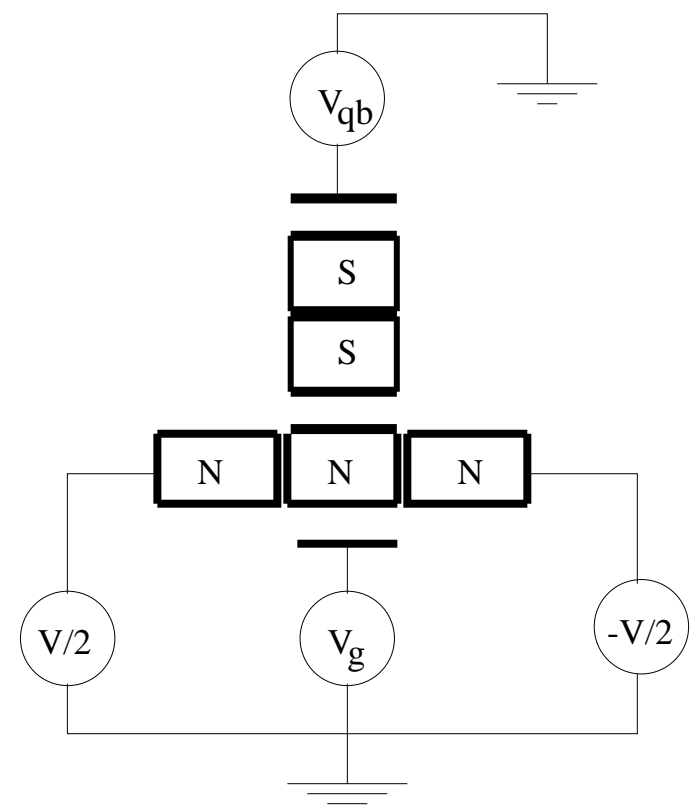

FIG. 1. The circuit consisting of a q-bit plus a SET transistor used as a measuring device.

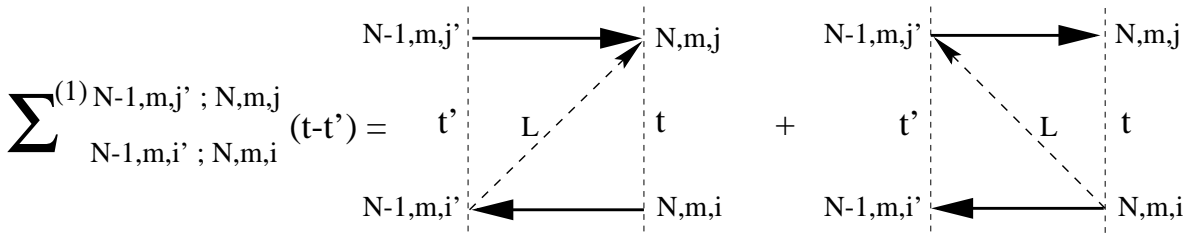

FIG. 2. The first order diagram for the transition rates. 
"ppp.dat"

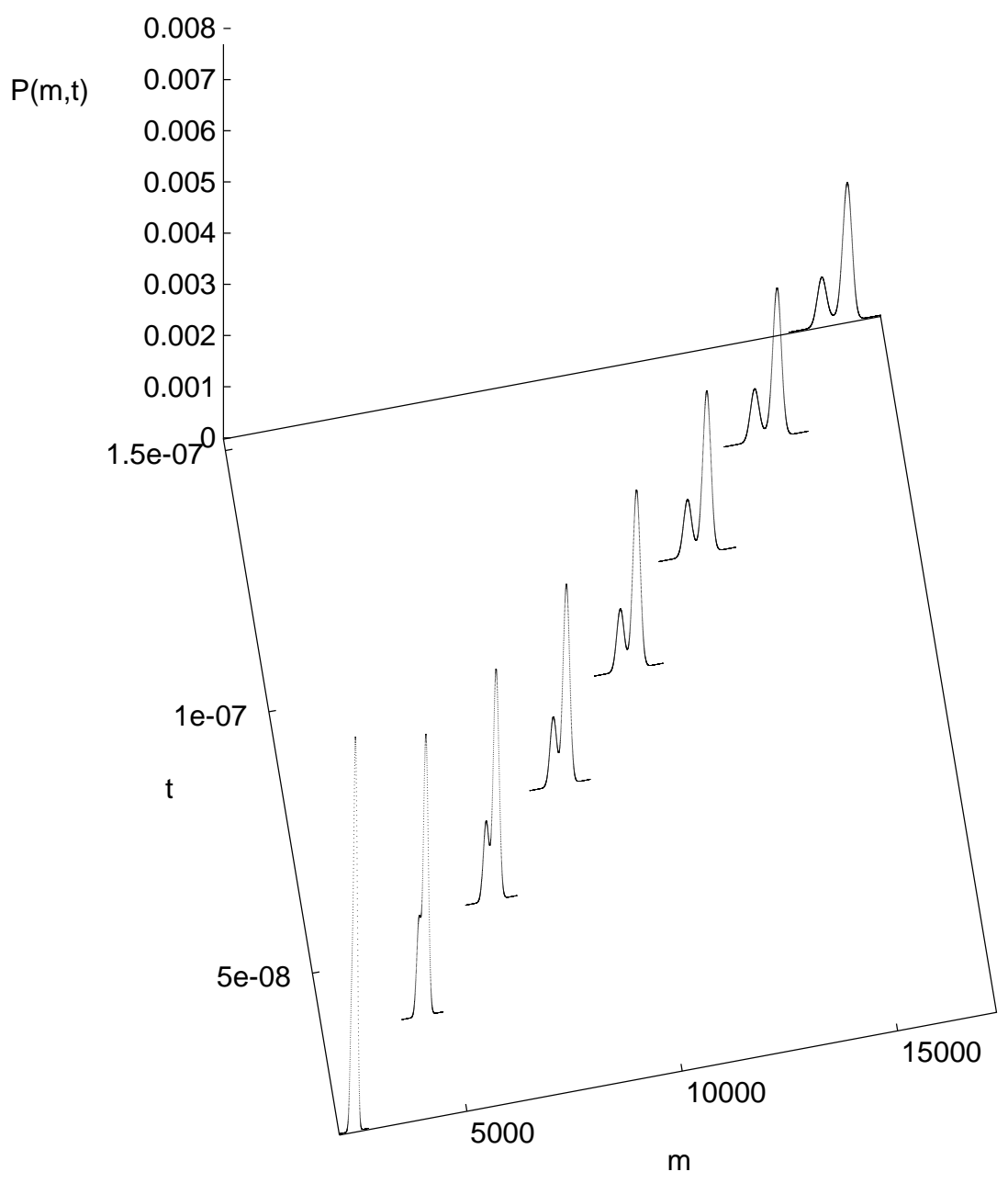

FIG. 3. $P(m, t)$, the probability that $m$ electrons have tunneled during time $t$. The parameters are those given in the text, $E_{\mathrm{J}}=0.1 \mathrm{~K}$. The time is measured in seconds. The initial amplitudes of the q-bit's states: $a=\sqrt{0.75}, b=\sqrt{0.25}$. 
"ppp.dat"

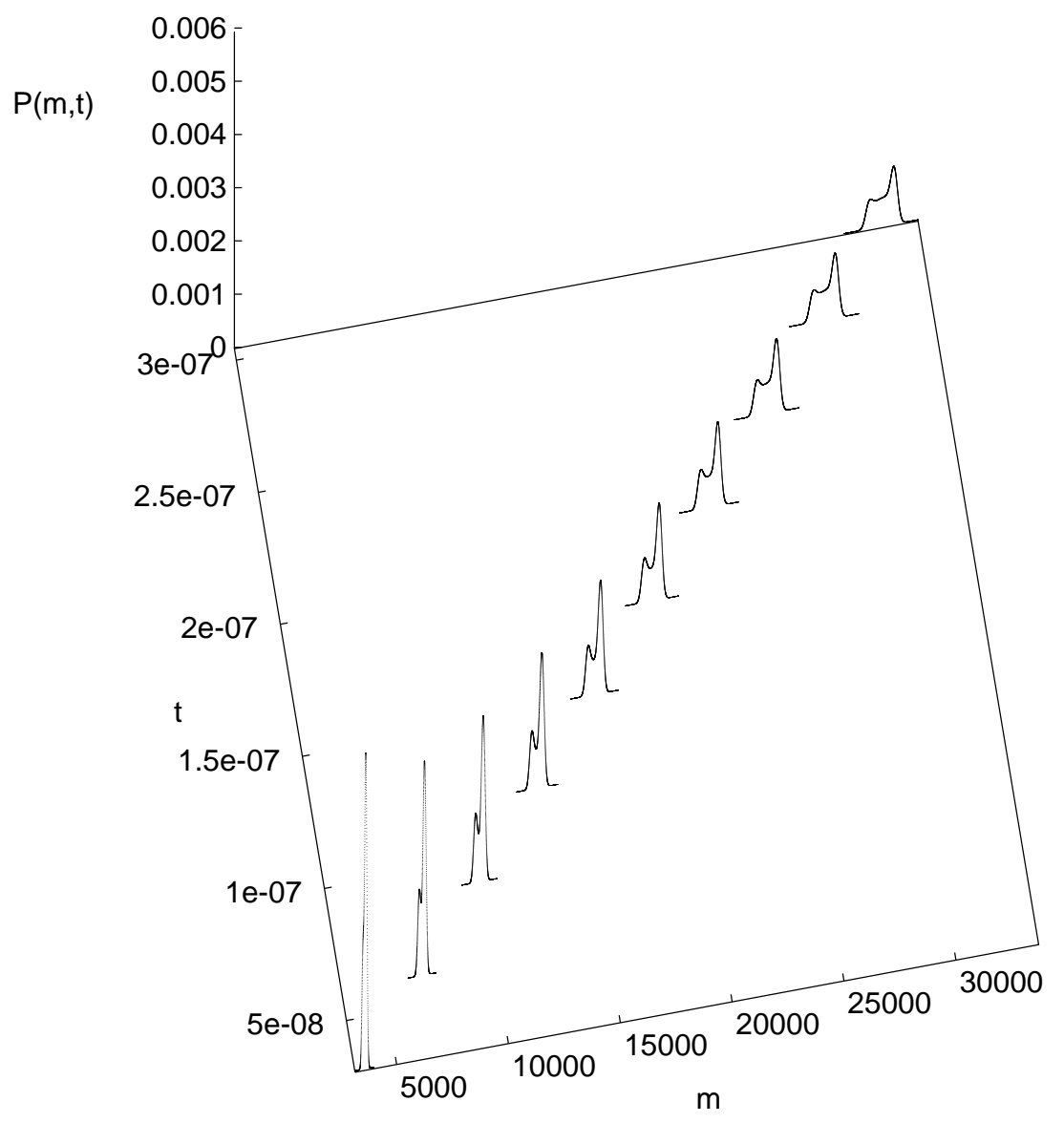

FIG. 4. $P(m, t)$, the probability that $m$ electrons have tunneled during time $t$. The parameters are those given in the text, $E_{\mathrm{J}}=0.25 \mathrm{~K}$. The time is measured in seconds. The initial amplitudes of the q-bit's states: $a=\sqrt{0.75}, b=\sqrt{0.25}$. 